\title{
Iron Metabolism: From Inflammation to Cancer
}

\author{
Di Paola A1, Tortora $C^{2}$, Argenziano $M^{2}$, Di Leva $C^{2}$ \\ and Rossi $\mathbf{F}^{2 *}$ \\ ${ }^{1}$ Department of Experimental Medicine, University \\ of Campania "Luigi Vanvitelli”, Via S Maria di \\ Costantinopoli, Italy \\ ${ }^{2}$ Department of Woman, Child and General and Specialist \\ Surgery, University of Campania "Luigi Vanvitelli”, Via L \\ De Crecchio, Italy \\ *Corresponding author: Rossi F, Prof., MD. \\ Department of Woman, Child and General and Special \\ Surgery, University of Campania “Luigi Vanvitelli” Via De \\ Crecchio, 4 - 80138 Naple, Italy
}

Received: April 11, 2021; Accepted: May 13, 2021; Published: May 20, 2021

\begin{abstract}
Iron is a trace element essential for several physiological cell functions and any alteration in its metabolism could be associated to the onset of several disorders. Cells normally avoid any dysregulation, activating fine molecular mechanisms to balance iron uptake, utilization, recycling, storage and export. The main "actors" in this event are hepcidin, ferroportin, ferritin and transferrin, both at cell and systemic level. Dysregulation in iron homeostasis is closely related to inflammation onset and perpetuation, osteoporosis and cancer progression. During inflammation, it has been observed a reduction in circulating iron as direct consequence of increase in ferritin levels, aimed to contain inflammatory processes and in many cases to restore the immune response. Iron overload directly promotes bone resorption and inhibits bone formation inducing osteoporosis. Moreover, iron cellular accumulation is responsible for ROS production with consequent DNA damage and neoplastic transformation of cells. In conclusion, even though many molecular mechanisms have to be clarified, targeting iron and also the mediators of its metabolism could be useful to manage a great variety of disorders, such inflammation, immune diseases, osteoporosis and cancer.
\end{abstract}

Keywords: Iron metabolism; Inflammation; Osteoporosis; Cancer; Ironrelated pathologies

\section{Introduction}

Iron is a very important trace element for organic systems, in particular in literature its vital role is well documented especially in mammal cells [1,2]. Iron is physiologically involved in several biological processes such DNA synthesis and repair, cellular metabolism and signaling [3,4], synthesis of hemoglobin, neurotransmission, cell growth and differentiation, immunity and others $[5,6]$. Considering the importance of iron in cell biology, a deregulation in its metabolism could damage cells, tissues and organ functions. For this reason, sophisticated molecular mechanisms are necessary to coordinate and maintain a proper homeostasis among iron uptake, utilization, recycling, storage and export [5,7]. Iron levels are normally equal to 3-4 gr with a daily loss of 1-2 mg [8] and these standard range is guaranteed by several key proteins: hepcidin, Ferroportin (FPN-1), ferritin and Transferrin (TF) are the principal regulators [9]. Hepcidin is a peptide hormone produced by liver and responsible for degradation and inhibition of FPN-1 [10]. FPNlis the only known iron exporter located on enterocyte basolateral membrane, where it mediates the dietary iron absorption as ferrous ion [11]. FPN-1 also limits the iron recycling from hepatocytes and macrophages, that are the cells principally involved in intracellular iron storage as ferritin $[12,13]$. TF is instead responsible for binding serum iron and delivering it to target cells expressing Transferrin Receptor 1 (TFR-1) that is triggered for the internalization of iron/ transferrin/TFR-1 complex [14]. In human beings systemic iron levels are principally controlled by hepcidin- FPN-1 axis [15], even though genetics and structure-function evaluations are ongoing to better clarify the exact molecular mechanism underlying their interaction [16-18]. Hepcidin is certainly the key protein in regulating iron levels and it could also serve as inflammatory marker [19]. During infection or inflammation its expression increases as consequence of pro-inflammatory cytokines production, especially of IL-6. Hepcidin binds FPN-1 inhibiting both bowel iron absorption and iron mobilization from hepatocytes and macrophages [20]. Therefore, during inflammation and infection processes, a protective condition known as "hypoferremia" develops to limit the vicious circle below inflammatory processes (iron-mediated activation of pro-inflammatory macrophages and further production of proinflammatory mediators [3]) and also to counteract the pathogens which metabolism depends on iron utilization. Dysregulation in iron metabolism is observed also in neurodegeneration [21] and cancer [22]. Iron indeed mediates the production of reactive oxygen species (ROS) by the Fenton reaction, thus inducing DNA damage, protein and lipid modifications, tumor microenvironment alteration and other events involved in tumor onset and progression [23-25]. Also in non-pathological conditions, the evaluation of ferritin and hepcidin levels could be useful indicator of circulating iron concentration. For example, during pregnancy hepcidin increases in the first trimester, while in the second and third ones it decreases principally to facilitate the absorption of dietary iron [26]. Biological systems normally work to avoid or compensate both excess and deficiency of iron. The excess is principally genetic (hereditary hemochromatosis, $\mathrm{HH}$ ), but it could be also related to poor erythropoiesis or to secondary iron overloading conditions (i.e. transfusion-dependent thalassemia) $[27,28]$. Similarly, iron deficiency could cause health problems, among them the most common is iron deficiency anemia $[5,29,30]$, but also cognitive complications in children [31-33]. Iron deficiency anemia is a group of sideropenic anemias characterized by low level of iron in plasma, low iron store, low transferrin saturation and other marks [32]. In the last decade, several authors described the involvement of iron and all the molecules responsible for its metabolism in pathogenesis of many other disorders, also extremely spread and burdensome for public health. In particular, this review has the aim to describe the 


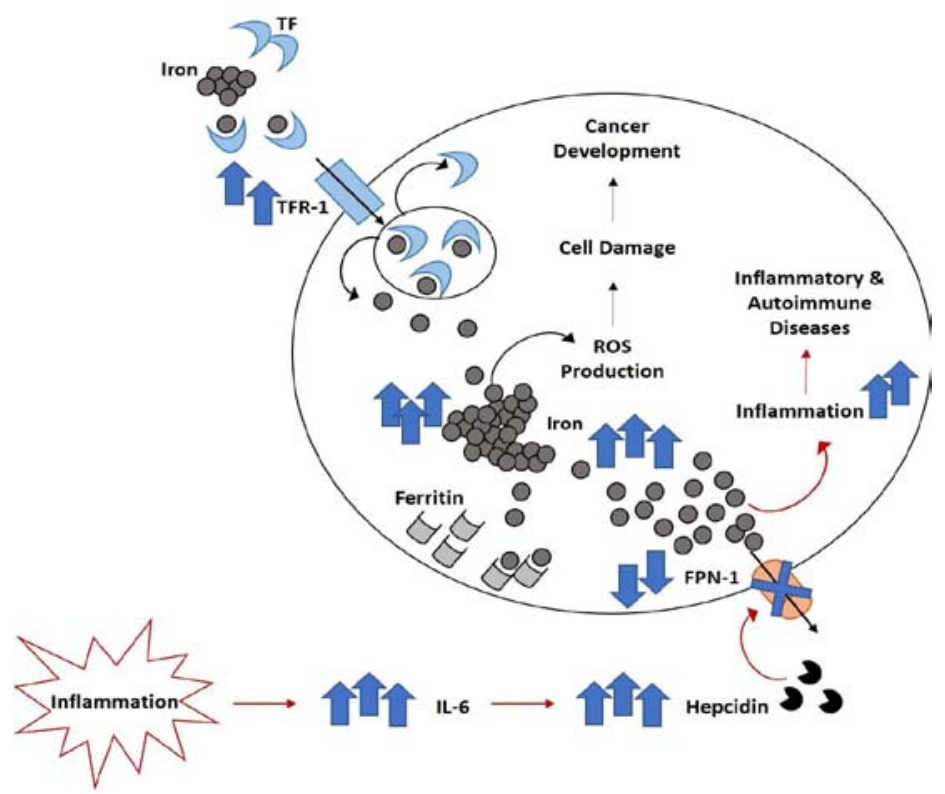

Figure 1: Iron metabolism alteration. Both systemic and cellular alteration in iron metabolism could be related to cancer development and onset of inflammatory diseases, including osteoporosis. TF: Transferrin; TFR-1: Transferrin Receptor 1; ROS: Reactive Oxygen Species; FPN-1: Ferroportin 1; IL-6: Interleukin-6.

involvement of iron metabolism in the pathogenesis of inflammation, osteoporosis and cancer, which represent the most common and diffuse health burden in humans (Figure 1).

\section{Iron Metabolism}

Iron levels are finely regulated at both systemic and cellular level. Both heme and non-heme dietary iron are present in the oxidized state $\left(\mathrm{Fe}^{3+}\right) . \mathrm{Fe}^{3+}$ binds to TF forming a complex that recognizes the TFR1 on the outer side of cell membrane. This complex is internalized in the endosome by endocytosis, and $\mathrm{Fe}^{3+}$ is reduced to $\mathrm{Fe}^{2+}$ by iron reductase, such as duodenal cytochrome b reductase (Dcytb), and transported into enterocytes by Divalent Metal Transporter 1 (DMT1) [34-37]. This metabolically active iron can be distributed to different cell compartments for different metabolic needs, or stored in ferritin, a protein that concentrates iron in an inactive form for later use [38]. The excess of iron can be exported out of the cell through a conserved multitransmembrane protein, FPN-1, the only known cellular iron efflux pomp which cooperates with ceruloplasmin or hephaestin to maintain cellular iron homeostasis [39]. At cellular level iron homeostasis is achieved by iron-regulatory proteins (IRP1 and IRP2). These proteins bind to Iron Responsive Elements (IREs) [40] and regulate the expression of proteins involved in iron import (TFR1, DMT1), storage (ferritin) and export (FPN-1) [41]. In a condition of iron deficiency, IRPs bind to $5^{\prime}$ IREs present in both ferritin and FPN-1 mRNAs to repress their translation, and to $3^{\prime}$ IREs in TFR-1 mRNA to prevent its degradation. Excess cytosolic iron destabilizes IRP1 and IRP2, preventing them from binding to IREs, resulting in increased synthesis of ferritin and FPN-1 and enhanced degradation of TFR-1 mRNA. At systemic level iron homeostasis is maintained by hepcidin, a circulating hormone that regulates iron levels inducing FPN-1 degradation [42].

\section{Iron and Inflammation}

It is widely known and well documented in literature that iron and inflammation are strongly related. When inflammation arises plasma iron concentrations decrease [43]. This response is called "hypoferremia of inflammation" and is driven by hepcidin that downregulates FPN-1 leading to an iron decrease into extracellular fluid from all its sources. In this way, iron is retained in macrophages of the liver and the spleen, and its absorption is decreased [44]. In accord, the hypoferremia of inflammation is absent in hepcidin knockout mice. The inflammatory increase in hepcidin levels is caused by increased concentrations of IL-6 (interleukin-6), that induces the transcription of the hepcidin gene through the JAK2/ STAT3 pathway [45].

During inflammation, ferritin not only reflects iron status but also acts as an acute phase reactant. It is plausible that high ferritin levels in diseases with high-grade inflammation could serve as regulatory mechanism to limit inflammation [46]. Evidences suggest that ferritin is able to reduce inflammation and to restore immunological response by binding to its receptor. Ferritin modulates the immune response by suppressing lymphocyte maturation, reducing the iron availability to lymphogenesis [47]. Moreover, ferritin downregulates the immune response due to activation of regulatory T-cells and synthesis of the antinflammatory IL-10. While many studies support this hypothesis, other data suggest a direct causal role of ferritin in mediating inflammatory responses in several rheumatologic, immunologic, malignant and infectious disorders. In these diseases ferritin could act as a local cytokine responsible for activation of inducible NO synthase and for the increased synthesis of IL- $1 \beta[46,48]$. Ruddel et al. investigated the role of ferritin in chronic liver injury and cirrhosis demonstrating that ferritin acts as a local cytokine inducing proinflammatory mediators activation [49]. Therefore, in addition to its homeostatic role, ferritin could be used as a biomarker of disease progress and prognosis as well as a target for therapeutic intervention.

Iron plays also a direct role in immune response by regulating macrophage function. In chronic inflammation and autoimmune 
diseases, macrophage iron retention seems to have an important pathogenetic role [50-52]. The intracellular iron of macrophages can modify their inflammatory response. Iron turnover is different in M1 and M2 cells. Due to a low expression of FPN-1 and Haemoxygenase 1 (HO-1), M1 macrophages are rich in ferritin and prone to iron accumulation, whereas M2 are able to metabolize and export iron, resulting in lower intracellular iron concentrations [53]. Macrophage iron levels can alter their polarization [54]. Low intracellular iron levels inhibit the expression of pro inflammatory cytokines, whereas increased levels induce a pro-inflammatory response [55-57]. Indeed, in chronic inflammation iron is mostly acquired by macrophages through erythrophagocytosis and the DMT1. Inflammatory stimuli lead macrophage iron retention also by down-regulating the expression of FPN-1 thus blocking the release of iron from these cells.

Moreover, inflammatory cytokines induce an increase of circulating hepcidin that consequently causes internalization and degradation of FPN-1 [58].

Accordingly, Zhang et al. showed that iron retention in macrophages lacking FPN-1 stimulates the expression of proinflammatory cytokines and the innate immune response in vivo [59].

Therefore, in chronic inflammation and autoimmune diseases, macrophage iron retention seems to have an important pathogenetic role. Macrophage polarization profile resulting from prolonged exposure to iron in tissue microenvironment, correlates with a proinflammatory (M1-like) phenotype that is associated with tissue damage in several inflammatory diseases [60-62]. It has been reported that iron overloading in macrophages occurs in human chronic venous leg ulcers causing a polarization toward a pro-inflammatory M1-like phenotype. Moreover, increased intracellular iron polarizes the macrophages toward a pro-inflammatory state in the injured spinal cord [60] and in chronic active multiple sclerosis. Also in a mouse model of sickle disease, macrophage iron accumulation induces a proinflammatory phenoytpe in hepatic macrophages [62].

Also in ITP, it has been observed a prevalence of M1 macrophages, responsible for an increased release of pro-inflammatory cytokines $[63,64]$. Accordingly, the switch from the M1 pro-inflammatory phenotype to the M2 anti-inflammatory type in ITP pediatric patients is correlated to a reduction of the inflammatory state and to a restoration of the immune system function [65]. In accordance with these findings, Pereira et al. demonstrated that acute iron deprivation in human macrophages causes anti-inflammatory responses. In detail, they demonstrated that Lipopolysaccharide (LPS) polarization of iron-deprived human macrophages induces a reduction of the proinflammatory cytokines Interleukin-1 $\beta$ (IL-1 $\beta$ ) and tumor necrosis factor (TNF- $\alpha$ ) suggesting that iron chelation could prevent LPS polarization [66].

Recently, it has been investigated the effect of tissue iron overload on atherosclerosis. Cornelissen et al. suggested that hepcidin could play different roles in atherogenesis depending about the stage of atherosclerosis. In early- to mid-stage plaques, inhibition of hepcidin may have beneficial effects containing the effects of pro-inflammatory macrophages, while in late stage lesions the reduced macrophage iron could induce plaque progression increasing angiogenesis, permeability, and inflammatory cells recruitment [67].
A dysregulated iron metabolism occurs also in chronic inflammatory conditions such as Inflammatory Bowel Disease (IBD), and leads to abnormal intracellular sequestration of iron and a decrease in circulating iron concentrations. The increased hepcidin levels leads to FPN-1 degradation decreasing iron concentrations with a consequent reduction in reactive oxygen species production and tissue damage [68]. However, persistent hypoferremia inevitably compromises erythropoiesis inducing a disorder known as the anemia of inflammation (AI) [69].

Iron deficiency also induces the production of FGF23, a bonesecreted hormone that acts as a central regulator of phosphate and vitamin D homeostasis, and bone mineralization [70]. A direct effect of iron deficiency on FGF 23 production was demonstrated by the ability of iron chelators to stimulate FGF23 production in isolated bone cell cultures [71,72]. The mechanisms for inflammatory-mediated FGF23 induction are still not clear, even though some studies suggest a role for Hypoxia-Inducible Factor-1 $\alpha$ (HIF-1 $\alpha$ ), NF- $\kappa B$ and IL-6 $[73,74]$. These findings are important in the setting of chronic inflammatory diseases which are characterized by elevated FGF23 levels.

Certainly, a better understanding of the role of iron in inflammatory conditions, macrophage polarization and autoimmunity could be useful to the development of innovative therapeutic strategies.

\section{Iron Chelation as Therapeutic Strategy in Inflammation}

Considering the importance of iron in both physiological and pathological events, targeting its molecular signaling pathways could represent a promising therapeutic strategy to improve the outcome of many others diseases. In particular, the chelation of iron by means of natural and synthetic compounds could influence the oxidative status, contrasting acute and chronic inflammatory processes. Among the natural products, one of the most known is the microbial chelator Desferal (or Deferoxamine, DFO), a siderophore normally produced by Streptomyces spp. to obtain from host the iron necessary for growth [75]. In literature, the evidences about its effectiveness as antiinflammatory agent are in contrast. Indeed, while it has been seen to reduce LPS-induced inflammation in mice, on the other hand DFO also promotes the infection of Yersinia enterocolitica or Candida albicans, maybe because of its own microbial origin [76,77]. Other important natural iron chelators with anti-inflammatory functions are the curcuminoids and African walnut extracts which are both able to reduce the toxic effects of iron overload in thalassemic mice [78]. The actual utility of these compounds in contrasting inflammation must to be better clarified. As regards the synthetic compounds, Kalinowski and Richardson already in 2007 described the effectiveness of Deferiprone (DFP) as bacteriostatic agent, since its capability to contrast iron overload and thus also inflammation and infection [79]. Another available synthetic iron chelator is DIBI that differently from DFO and DFP, the two well-known hematological chelators described above, was designed just as anti-inflammatory agent [80]. Indeed, it is more effective in its therapeutic activity, because it not only binds iron, but it provides compartmentalized sinks for iron thus reducing its availability in inflammatory reactions $[76,81]$. Although it is evident the potential benefit in using iron chelators in inflammatory conditions, there are still many doubts principally due to the medical assumption that stored iron is safe. In this point of view, to treat an 
inflammatory state by the induction of a second disorder, namely the deprivation of "safe iron", could be controversial [82].

\section{Iron Metabolism and Osteoporosis}

Iron homeostasis strongly affects bone metabolism [83]. Several diseases such as hemochromatosis, hemosiderosis, ß-thalassemia, sickle cell disease and liver diseases characterized by iron overload are frequently accompanied by Osteoporosis (OP) [84,85]. Evidences suggest that both iron deficiency and iron overload negatively affect bone, acting directly on bone cells. Iron deficiency seems to reduce Bone Mineral Density (BMD) causing a severe alteration of bone structure. Iron overload directly promotes bone resorption and inhibits bone formation inducing osteopenia and OP [86]. Excess iron increases osteoclastogenesis and bone resorption [87] while leads to a significant reduction in osteoblast differentiation as revealed by decreased mineralization and expression of osteogenic genes [88]. Bone loss caused by iron accumulation is also associated with the apoptosis of Bone Marrow Mesenchymal Stem Cells (BMSCs) capable of differentiating into osteoblasts [89].

Therefore, the reduction of iron levels is suggested to be a potential therapeutic approach for OP treatment. The iron chelators are the commonly used drugs to treat iron-overload diseases by promoting iron excretion and reducing accumulated iron in tissues [90]. Three iron chelators have been approved for clinical use: DFO, DFP and Deferasirox (DFX). DFO is the first approved iron chelator and is used by subcutaneous or intravenous injection. DFP and DFX are oral iron chelators. All these iron chelators are shown to be effective in chelating iron from the heart and liver [91]. Several in vitro and in vivo studies demonstrated how iron overload alters bone balance. Iron overload-induced OP in zebrafish can be obtained by adding Ferric Ammonium Citrate (FAC) in the fish water. FAC causes osteogenic inhibition, as revealed by a decrease in bone calcification and cartilage development in both larval and adult fish [90,92]. Iron accumulation in bone is associated with bone loss in ovariectomized rats, and iron chelation partially prevents bone loss [93].

Iron overload is a risk factor for progressive bone loss in healthy postmenopausal women and middle-aged men [94]. In 2014, Rossi et al demonstrated that iron overload causes overactivity of Osteoclasts (OCs) in $\beta$ Thalassemia Major (TM) patients inducing activation of the Transient Receptor Potential Vanilloid Type 1 (TRPV1) channels suggesting that a chelation therapy could have a role in alleviating TM-associated OP [95]. In accordance, a few years later, they tested Eltrombopag (ELT), an agonist at Thrombopoietin receptor with emerging chelating properties, in combination with DFX in iron overloaded OCs from thalassemic patients demonstrating, for the first time, that ELT is able to reduce bone mass loss [28].

Recent studies suggested that hepcidin, the major regulator of iron metabolism, is involved in OP [96-98]. Hepcidin knockout mice had a higher serum ferritin level and higher iron in the liver than controls and showed low bone mass and changes in bone microarchitecture [99]. Liu et al. compared serum hepcidin levels in 40 patients with $\mathrm{OP}$ and 40 healthy controls demonstrating lower serum hepcidin levels and higher iron levels in patients with OP and that the serum hepcidin level was negatively related to the serum iron level [100]. In contrast, Sato et al. demonstrated that the serum hepcidin level was positively related to serum iron and ferritin levels, and serum iron levels were positively related to BMD [99]. These studies suggest hepcidin as a possible useful therapeutic target in OP.

\section{Iron Metabolism and Cancer}

Iron metabolism dysregulation, in particular the accumulation of iron into cells, is responsible for the onset of cancer [3,24,25,101103], contributing to tumorigenesis, tumor progression, metastasis and tumor microenvironment (TME) alteration [3,25,103]. High intracellular iron concentration is closely related to tumor; indeed, cancer cells are more dependent on iron for their growth and proliferation than healthy cells $[102,104,105]$.

Proteins involved in regulation of iron metabolism show an alteration of their expression and activity in cancer cells [24], for example, TFR-1 is present at very high levels in several solid tumors $[102,106-108]$ and in leukemia [105]. This overexpression results in an increase in intracellular iron concentration and consequently in ROS production contributing not only to tumor growth and survival in the early stages of cancer, but also to metastasis development in the late stages [102,105-107,109]. It has been reported that also STEAP (human 6-transmembrane epithelial antigen of prostate) proteins show an increased expression in breast, prostate, pancreatic cancers and in acute leukemia, promoting tumor progression and invasion capacity $[24,102,105]$. Another important protein involved in an increased iron storage in cancer cells is ferritin; its over-expression is detected in different tumors, such as glioblastoma multiforme, breast, prostate and pancreatic cancers $[108,110,111]$. Also patients with Acute Myeloid Leukemia (AML) show an increase in ferritin levels which contribute to leukemia cells proliferation and drug resistance $[47,105,112]$. However, FPN-1 and hepcidin are the major constituents of iron regulatory pathways primarily involved in cancer development and metastasis [108]. FPN-1 is downregulated in patients with breast, prostate and hepatocellular cancers, thus contributing to its accumulation into cells and consequently to increase tumor growth $[103,108,110]$. In particular, Pinnix et al. demonstrated that human breast cancer tissue expressed low levels of FPN-1 compared to the human breast normal tissue and that this reduction of FPN-1 was associated to a poor prognosis [113]. Transfection of breast cancer cells with FPN-1 reduced their growth and proliferation, suggesting that FPN-1 could be a predictor of prognosis in breast cancer [113]. The reduction of FPN-1 levels has been observed also in AML cell lines [105,114], but it is associated with good prognosis, maybe due to an increased sensitivity to chemotherapy $[105,115]$. Also hepcidin expression is altered in several tumors [108]. It is known that hepcidin levels increase both during inflammation [116-118] and in cancer patients [108], maybe due to the inflammatory state of cancer tissue [119]. In particular, hepcidin is present at very high levels in breast cancer, prostate cancer and multiple myeloma [120-122]. It exhibits pro-oncogenic property by inducing internalization and degradation of FPN-1 with a consequent increase of iron concentration [108]. Also in acute leukemia it is reported an increase of hepcidin levels, which causes iron accumulation in cells thus contributing to tumor progression and growth [105]. Moreover, in addition to the increased levels of systemic hepcidin, also cancer cells themselves produce high levels of hepcidin which acts locally inducing FPN-1 degradation $[105,113,123]$. High levels of hepcidin is related to poor prognosis 
and metastatic progression $[113,124]$.

In conclusion, dysregulation of iron metabolism is closely related to cancer development and progression [3]. Iron accumulation into cells determines ROS production which causes DNA damage and cellular transformation within tumor cells [102,125]. Targeting iron with iron chelator could be useful to contain tumor progression $[2,6]$. Several studies demonstrated that iron chelation inhibits proliferation and induces apoptosis in both hematological and solid tumors [24,105,126-129], representing a novel and effective therapeutic anticancer strategy.

\section{Iron Metabolism and Tumor Microenvironment}

Iron metabolism is altered not only in cancer cells, but also in cells of Tumor Microenvironment (TME) [103,130]. TME refers to the cellular and extracellular environment surrounding the tumor [131]. It includes blood vessels, signaling molecules and cytokines, extracellular matrix, other non-malignant cells such as lymphocytes and Tumor-Associated Macrophages (TAMs) [25,131,132]. TAMs could be present in two different activation states: the classically activated macrophages (M1) and the alternatively activated Macrophages (M2) [132]. Considering that macrophages are immune cells characterized by an elevated plasticity, it is reported that different states of macrophages activation are contemporary present and that macrophages adopt a specific phenotype in response to different microenvironment stimuli [25,133]. M1 macrophages are characterized by an elevated iron content which is responsible for inducing inflammation and inhibiting pathogen growth. They show anti-microbial, pro-inflammatory and anti-tumor properties, by releasing high levels of pro-inflammatory cytokines, such as IL-6, TNF- $\alpha$, IL-1 $\beta$ and Nitric Oxide Synthase (iNOS) $[65,132]$ In contrast, M2 macrophages release high quantity of iron, thus contributing to cell proliferation. They have both anti-inflammatory and immunosuppressive functions, by releasing anti-inflammatory cytokines, such as IL-10 and Transforming Growth Factor- $\beta$ (TGF- $\beta$ ), and also pro-angiogenic and pro-fibrotic properties, contributing to tumor progression $[65,132]$. Therefore, while pro-inflammatory M1 macrophages are responsible for iron uptake and storage with a consequent iron retention; anti-inflammatory M2 phenotype is involved in iron release in the extracellular space $[103,132]$. Recalcati et al. demonstrated that M2 macrophages are characterized by increased levels of FPN-1 and reduced levels of ferritin [134], determining iron release into the surrounding microenvironment. This macrophage phenotype determines tumor progression and growth by releasing high levels of iron, which could affect the metabolism of cancer cells $[103,130,135]$.

\section{Iron Metabolism Involvement in Cancer Metastasis and Angiogenesis}

Intracellular iron accumulation results in an increased ROS production which are responsible for local microenvironment acidification thus contributing to cell neo-vascularization, invasion and migration $[102,103,136]$. In literature it is reported that iron overload induces a reduction in Vascular Endothelial Growth Factor (VEGF) expression, inhibiting cell proliferation and invasion, while its reduction determines an increase in VEGF expression, through stabilization of HIF-1 [24]. In contrast, several authors also reported that HO-1, degrading heme into biliverdin, carbon monoxide and iron, contributes to iron accumulation [24], thus promoting VEGF expression with consequent metastasis and angiogenesis [102]. In accordance, HO-1 overactivation in breast cancer induces an accumulation of iron causing cells migration and invasion [136,137]. In prostate and in lung cancers an increase in intracellular iron levels contributes to cell migration and metastatic spread by hepcidininduced FPN-1 degradation and iron overload with ferrous sulfate, respectively $[138,139]$. Iron is also able to activate metalloproteases, thus contributing to cancer cells invasion and migration [102]. In particular, it has been demonstrated that iron overload promotes matrix metallopeptidase 9 (MMP-9) activation in head and neck squamous carcinoma, through MAPK and AKT pathways activation, thus contributing to cancer cell invasion and metastasis [140].

\section{Iron Metabolism and Cell Cycle}

Iron has also a key role in regulation of cell cycle. In G1 phase cells have an increase request of iron for DNA synthesis [24,25]. In particular, the ribonucleotide reductase, whose activity is closely dependent from iron, plays an important role in DNA synthesis and is responsible for cell viability [24]. Several studies are based on the use of iron chelators as anti-cancer drug to inhibit the activity of this enzyme [24,141]. Also many DNA polymerases are dependent on iron for their activity; they contain an iron-sulphur cluster which are necessary to DNA synthesis $[24,142]$.

Finally, iron modulates the activity of proteins involved in cell cycle progression, cyclins and cyclins-dependent kinases [24,25,143]. It has been demonstrated that iron chelation could determine a cell cycle arrest in G1/S phase reducing cyclins expression, thus contributing to block cell cycle progression $[24,25,144]$.

\section{Iron Metabolism and Hypoxia}

Hypoxia is a condition of decreased oxygen availability, responsible for tumor progression and therapy resistance $[145,146]$. HIFs are alpha/beta heterodimeric transcription factors with a key role in hypoxia responses both in normal and cancer cells $[146,147]$. In condition of normoxia, HIFs activity is finely regulated by the Prolyl-Hydroxylase Domain enzymes (PHDs) which mediate their degradation [147]. During hypoxia, PHDs are inhibited and the a subunits can form heterodimers with $\beta$ subunits which migrate into nucleus and activate transcription of several genes involved in cellular stress, angiogenesis, apoptosis [147].

It is reported that HIFs activity is increased in several tumors and its overexpression is related to iron accumulation and consequent cancer cell proliferation [24]. There is a crosstalk between oxygen and iron metabolisms mainly mediated by HIFs [147]. In particular, HIF1 promotes TFR-1 expression inducing an increase of iron uptake $[24,147]$; it also causes HO-1 activation, thus contributing to iron accumulation in cells $[24,147]$. Finally, it is responsible for iron oxidation by ceruloplasmin, promoting transferrin-mediated iron sequestration and, consequently, its internalization [24,147]. Also, HIF2 is involved in regulation of iron metabolism: it determines an increase of iron accumulation in enterocytes, by activating the transcription of DMT1, FPN-1 and duodenal cytochrome [24,147]. Xue et al. have demonstrated that in colon cancer HIF2 promotes 
DMT1 overexpression with a consequent increase of intracellular iron concentration, thus inducing cancer progression [148].

\section{Iron Metabolism as Therapeutic Target in Cancer}

Iron deprivation has indisputable beneficial effects in contrasting tumor growth and progression. Cancer cells need more iron than healthy cells, because of their high rate in DNA synthesis for example [149]. For this reason, they show higher levels of TFR-1 and lower levels of iron exporters [113]. Therefore, neoplastic cells are more sensitive to iron deprivation than normal cells and consequently may be susceptible to treatment with iron chelators [150]. DFO was the first iron chelator taken forward as anticancer drug for clinical trials already in 1995. It was used in a Phase II trial on neuroblastoma patients observing a reduction in bone marrow infiltration and in tumor mass [151]. Although its efficacy, the use of DFO as anticancer drug presents several limitations: great variability in response, poor lipophilicity, rapid clearance and also the necessity of continuous infusions [152]. Amano et al. showed that the iron chelator DFX exerts its antineoplastic property in gastric cancer [153] and also in pancreatic cancer by inhibiting cell invasion capacity [154]. In contrast, a recent study demonstrated that both iron chelators ELT and DFX do not exert anti-cancer activity in osteosarcoma [101]. Moreover, it has been proposed the use of iron chelators as anti-cancer drugs also in leukemia $[105,155]$ by inhibiting cell proliferation and inducing apoptosis $[105,127,156]$. Also the iron chelator Dp44mt suppresses proliferation of several cancer cells, such as pancreatic cancer cells and glioma $[157,158]$. The anticancer role of thiosemicarbazone chelators also emerged and was mostly attributed to their capability to inhibit ribonucleotide reductase [159]. This kind of compounds had more experimental success in blood cancers than in solid ones [160]. Nowadays many innovative kinds of iron chelators are being taken into account as potential cancer therapy, such VLX600 that shows limited side effects and is capable to target both senescent and proliferative cells [161]. Then there are also several natural compounds for which anticancer effect have been recognized: silibinin, quercetin and epigallocatechin gallate suggested as chemopreventative agents [162-164], but not without any limitation such the bioavailability. The combination of iron chelators with chemotherapy is well documented in literature. For example, DFX, in combination with doxorubicin, cisplatin, and carboplatin is able to suppress cell growth and to induce apoptosis in breast cancer and other cancer types $[165,166]$, as well as the combination with gemcitabine blocks pancreatic cancer cell proliferation [128].

Certainly, the research in this field is still open and to explore the underlying mechanisms of action behind iron chelation in cancer could represent a great contribute in the clinical practice.

\section{Conclusions}

A well-balanced iron metabolism has a key role in maintaining the integrity of biological systems and functions, considering that iron is essential in sustaining molecular processes such DNA synthesis and repair, cellular metabolism and signaling, neurotransmission and also cell proliferation, growth and differentiation. The molecules principally involved in modulating the balance among iron uptake, utilization, recycling, storage and export are hepcidin, ferroportin, ferritin and transferrin. Even though their interactions must to be better characterized, it is known that they are finely regulated and that any alteration could contribute to onset and progression of cognitive complications, iron deficiency anemia, inflammation, osteoporosis, infection and cancer. During inflammation iron availability decreases as defense mechanism aimed to avoid the inflammatory processes normally powered by iron. This ion is indeed responsible for activation of pro-inflammatory macrophages as well as for the production of ROS that exacerbate the cell damage. Moreover, iron overload directly promotes bone resorption and inhibits bone formation inducing osteoporosis. In the same way, the intracellular iron accumulation could cause neoplastic cell transformation and also the malignant alteration of tumor microenvironment. All these evidences encourage to target iron and molecules involved in its metabolism to counteract inflammation, osteoporosis and cancer progression.

\section{References}

1. Rao R, Georgieff MK. Iron in fetal and neonatal nutrition. Semin Fetal Neonatal Med. 2007; 12: 54-63

2. Poetini MR, Musachio EAS, Araujo SM, Almeida FP, Dahleh MMM, Bortolotto VC, et al. Iron overload during the embryonic period develops hyperactive like behavior and dysregulation of biogenic amines in Drosophila melanogaster. Dev Biol. 2021; 475: 80-90.

3. Shen L, Zhou Y, He H, Chen W, Lenahan C, Li X, et al. Crosstalk between Macrophages, T Cells, and Iron Metabolism in Tumor Microenvironment. Oxidative medicine and cellular longevity. 2021; 2021: 8865791.

4. Torti SV, Manz DH, Paul BT, Blanchette-Farra N, Torti FM. Iron and Cancer. Annu Rev Nutr. 2018; 38: 97-125.

5. Benson CS, Shah A, Stanworth SJ, Frise CJ, Spiby H, Lax SJ, et al. The effect of iron deficiency and anaemia on women's health. Anaesthesia. 2021; 76: 84-95

6. Litton E, Lim J. Iron Metabolism: An Emerging Therapeutic Target in Critical Illness. Crit Care. 2019; 23: 81.

7. Andrews NC, Schmidt PJ. Iron homeostasis. Annu Rev Physiol. 2007; 69: 69-85.

8. Munoz M, Laso-Morales MJ, Gomez-Ramirez S, Cadellas M, Nunez-Matas MJ, Garcia-Erce JA. Pre-operative haemoglobin levels and iron status in a large multicentre cohort of patients undergoing major elective surgery. Anaesthesia. 2017; 72: 826-834.

9. Camaschella C, Nai A, Silvestri L. Iron metabolism and iron disorders revisited in the hepcidin era. Haematologica. 2020; 105: 260-272.

10. Nemeth E, Tuttle MS, Powelson J, Vaughn MB, Donovan A, Ward DM, et al. Hepcidin regulates cellular iron efflux by binding to ferroportin and inducing its internalization. Science. 2004; 306: 2090-2093.

11. Knutson MD, Oukka M, Koss LM, Aydemir F, Wessling-Resnick M. Iron release from macrophages after erythrophagocytosis is up-regulated by ferroportin 1 overexpression and down-regulated by hepcidin. Proc Natl Acad Sci U S A. 2005; 102: 1324-1328.

12. Daher R, Manceau H, Karim Z. Iron metabolism and the role of the ironregulating hormone hepcidin in health and disease. Presse medicale. 2017; 46: e272-e278.

13. Ward DM, Kaplan J. Ferroportin-mediated iron transport: expression and regulation. Biochim Biophys Acta. 2012; 1823: 1426-1433.

14. Karaskova E, Pospisilova D, Velganova-Veghova M, Geryk M, Volejnikova J, Holub D, et al. Importance of Hepcidin in the Etiopathogenesis of Anemia in Inflammatory Bowel Disease. Dig Dis Sci. 2020.

15. Ganz T. Systemic iron homeostasis. Physiol Rev. 2013; 93: 1721-1741.

16. Billesbolle CB, Azumaya CM, Kretsch RC, Powers AS, Gonen S, Schneider $S$, et al. Structure of hepcidin-bound ferroportin reveals iron homeostatic 
mechanisms. Nature. 2020; 58: 807-811.

17. Bonaccorsi di Patti MC, Polticelli F, Cece G, Cutone A, Felici F, Persichini $\mathrm{T}$, et al. A structural model of human ferroportin and of its iron binding site. FEBS J. 2014; 281: 2851-2860.

18. Vlasveld LT, Janssen R, Bardou-Jacquet E, Venselaar H, Hamdi-Roze $H$ Drakesmith $\mathrm{H}$, et al. Twenty Years of Ferroportin Disease: A Review or An Update of Published Clinical, Biochemical, Molecular, and Functional Features. Pharmaceuticals (Basel). 2019; 12.

19. Krawiec P, Mroczkowska-Juchkiewicz A, Pac-Kozuchowska E. Serum Hepcidin in Children with Inflammatory Bowel Disease. Inflamm Bowel Dis. 2017; 23: 2165-2171.

20. Casu C, Nemeth E, Rivella S. Hepcidin agonists as therapeutic tools. Blood 2018; 131: 1790-1794.

21. Ward RJ, Zucca FA, Duyn JH, Crichton RR, Zecca L. The role of iron in brain ageing and neurodegenerative disorders. Lancet Neurol. 2014; 13 1045-1060.

22. Dixon SJ, Stockwell BR. The role of iron and reactive oxygen species in cell death. Nat Chem Biol. 2014; 10: 9-17.

23. Kim MJ, Yun GJ, Kim SE. Metabolic Regulation of Ferroptosis in Cancer. Biology (Basel). 2021; 10: 83.

24. Torti SV, Torti FM. Iron and cancer: more ore to be mined. Nature reviews Cancer. 2013; 13: 342-355.

25. Wang Y, Yu L, Ding J, Chen Y. Iron Metabolism in Cancer. International journal of molecular sciences. 2019; 20.

26. Sangkhae V, Fisher AL, Chua KJ, Ruchala P, Ganz T, Nemeth E. Maternal hepcidin determines embryo iron homeostasis in mice. Blood. 2020; 136 2206-2216.

27. Pietrangelo A. Iron and the liver. Liver Int. 2016; 36: 116-123.

28. Punzo F, Tortora C, Argenziano M, Casale M, Perrotta S, Rossi F. Iron chelating properties of Eltrombopag: Investigating its role in thalassemiainduced osteoporosis. PLoS One. 2018; 13: e0208102.

29. Muckenthaler MU, Rivella S, Hentze MW, Galy B. A Red Carpet for Iron Metabolism. Cell. 2017; 168: 344-361.

30. Pratt JJ, Khan KS. Non-anaemic iron deficiency - a disease looking for recognition of diagnosis: a systematic review. Eur J Haematol. 2016; 96 618-628.

31. Andro M, Le Squere P, Estivin S, Gentric A. Anaemia and cognitive performances in the elderly: a systematic review. Eur J Neurol. 2013; 20 1234-1240.

32. Camaschella C. Iron-Deficiency Anemia. N Engl J Med. 2015; 373: 485-486

33. Haas JD, Brownlie Tt. Iron deficiency and reduced work capacity: a critical review of the research to determine a causal relationship. J Nutr. 2001; 131 676S-88S; discussion 88S-90S.

34. Bogdan AR, Miyazawa M, Hashimoto K, Tsuji Y. Regulators of Iron Homeostasis: New Players in Metabolism, Cell Death, and Disease. Trends Biochem Sci. 2016; 41: 274-286.

35. Milto IV, Suhodolo IV, Prokopieva VD, Klimenteva TK. Molecular and Cellular Bases of Iron Metabolism in Humans. Biochemistry (Mosc). 2016; 81: 549-564.

36. Munoz M, Villar I, Garcia-Erce JA. An update on iron physiology. World J Gastroenterol. 2009; 15: 4617-4626.

37. Wang J, Pantopoulos K. Regulation of cellular iron metabolism. Biochem J. 2011; 434: 365-381.

38. Lv H, Shang P. The significance, trafficking and determination of labile iron in cytosol, mitochondria and lysosomes. Metallomics. 2018; 10: 899-916.

39. Fuqua BK, Lu Y, Frazer DM, Darshan D, Wilkins SJ, Dunn L, et al. Severe Iron Metabolism Defects in Mice With Double Knockout of the Multicopper Ferroxidases Hephaestin and Ceruloplasmin. Cell Mol Gastroenterol
Hepatol. 2018; 6: 405-427.

40. Wilkinson N, Pantopoulos K. The IRP/IRE system in vivo: insights from mouse models. Front Pharmacol. 2014; 5: 176.

41. Anderson CP, Shen M, Eisenstein RS, Leibold EA. Mammalian iron metabolism and its control by iron regulatory proteins. Biochim Biophys Acta. 2012; 1823: 1468-1483.

42. Rishi G, Wallace DF, Subramaniam VN. Hepcidin: regulation of the master iron regulator. Bioscience reports. 2015; 35.

43. Ganz T. Iron and infection. Int J Hematol. 2018; 107: 7-15.

44. Ganz T. Anemia of Inflammation. N Engl J Med. 2019; 381: 1148-1157.

45. Sangkhae $\mathrm{V}$, Nemeth $\mathrm{E}$. To induce or not to induce: the fight over hepcidin regulation. Haematologica. 2019; 104: 1093-1095.

46. Kernan KF, Carcillo JA. Hyperferritinemia and inflammation. Int Immunol. 2017; 29: 401-409.

47. Broxmeyer HE, Williams DE, Geissler K, Hangoc G, Cooper S, Bicknell DC, et al. Suppressive effects in vivo of purified recombinant human $\mathrm{H}$-subunit (acidic) ferritin on murine myelopoiesis. Blood. 1989; 73: 74-79.

48. Giemza-Stoklosa J, Islam MA, Kotyla PJ. Hyperferritinaemia: An Iron Sword of Autoimmunity. Curr Pharm Des. 2019; 25: 2909-2918.

49. Ruddell RG, Hoang-Le D, Barwood JM, Rutherford PS, Piva TJ, Watters DJ, et al. Ferritin functions as a proinflammatory cytokine via iron-independent protein kinase $\mathrm{C}$ zeta/nuclear factor kappaB-regulated signaling in rat hepatic stellate cells. Hepatology. 2009; 49: 887-900.

50. Martinez FO, Helming L, Gordon S. Alternative activation of macrophages: an immunologic functional perspective. Annu Rev Immunol. 2009; 27: 451 483.

51. Mosser DM, Edwards JP. Exploring the full spectrum of macrophage activation. Nat Rev Immunol. 2008; 8: 958-969.

52. Recalcati S, Locati M, Gammella E, Invernizzi P, Cairo G. Iron levels in polarized macrophages: regulation of immunity and autoimmunity. Autoimmun Rev. 2012; 11: 883-889.

53. Boytard L, Spear R, Chinetti-Gbaguidi G, Acosta-Martin AE, Vanhoutte J, Lamblin $\mathrm{N}$, et al. Role of proinflammatory CD68(+) mannose receptor(-) macrophages in peroxiredoxin-1 expression and in abdominal aortic aneurysms in humans. Arterioscler Thromb Vasc Biol. 2013; 33: 431-438.

54. Handa P, Thomas S, Morgan-Stevenson V, Maliken BD, Gochanour E, Boukhar S, et al. Iron alters macrophage polarization status and leads to steatohepatitis and fibrogenesis. J Leukoc Biol. 2019; 105: 1015-1026.

55. She H, Xiong S, Lin M, Zandi E, Giulivi C, Tsukamoto H. Iron activates NFkappaB in Kupffer cells. Am J Physiol Gastrointest Liver Physiol. 2002; 283 . G719-G726.

56. Wang L, Harrington L, Trebicka E, Shi HN, Kagan JC, Hong CC, et al. Selective modulation of TLR4-activated inflammatory responses by altered iron homeostasis in mice. J Clin Invest. 2009; 119: 3322-3328.

57. Wang L, Johnson EE, Shi HN, Walker WA, Wessling-Resnick M, Cherayil BJ. Attenuated inflammatory responses in hemochromatosis reveal a role for iron in the regulation of macrophage cytokine translation. J Immunol. 2008; 181: $2723-2731$.

58. Ganz T, Nemeth E. Iron homeostasis in host defence and inflammation. Nat Rev Immunol. 2015; 15: 500-510.

59. Zhang Z, Zhang F, An P, Guo X, Shen Y, Tao Y, et al. Ferroportin1 deficiency in mouse macrophages impairs iron homeostasis and inflammatory responses. Blood. 2011; 118: 1912-1922.

60. Kroner A, Greenhalgh AD, Zarruk JG, Passos Dos Santos R, Gaestel M, David S. TNF and increased intracellular iron alter macrophage polarization to a detrimental M1 phenotype in the injured spinal cord. Neuron. 2014; 83: 1098-1116.

61. Sindrilaru A, Peters T, Wieschalka S, Baican C, Baican A, Peter $\mathrm{H}$, et al. An unrestrained proinflammatory M1 macrophage population induced by iron 
impairs wound healing in humans and mice. J Clin Invest. 2011; 121: 985997.

62. Vinchi F, Costa da Silva M, Ingoglia G, Petrillo S, Brinkman N, Zuercher A et al. Hemopexin therapy reverts heme-induced proinflammatory phenotypic switching of macrophages in a mouse model of sickle cell disease. Blood 2016; 127: 473-486.

63. Feng Q, Xu M, Yu YY, Hou Y, Mi X, Sun YX, et al. High-dose dexamethasone or all-trans-retinoic acid restores the balance of macrophages towards M2 in immune thrombocytopenia. J Thromb Haemost. 2017; 15: 1845-1858.

64. Xu P, Jiang Y, Zuo H, Liu X, Xia T, Zhou R, et al. Vincristine-loaded platelets coated with anti-CD41 mAbs: a new macrophage targeting proposal for the treatment of immune thrombocytopenia. Biomater Sci. 2019; 7: 4568-4577.

65. Di Paola A, Palumbo G, Merli P, Argenziano M, Tortora C, Strocchio L, et al. Effects of Eltrombopag on In Vitro Macrophage Polarization in Pediatric Immune Thrombocytopenia. Int J Mol Sci. 2020; 22: 97.

66. Pereira M, Chen TD, Buang N, Olona A, Ko JH, Prendecki M, et al. Acute Iron Deprivation Reprograms Human Macrophage Metabolism and Reduces Inflammation In Vivo. Cell Rep. 2019; 28: 498-511.e5.

67. Cornelissen A, Guo L, Sakamoto A, Virmani R, Finn AV. New insights into the role of iron in inflammation and atherosclerosis. EBioMedicine. 2019; 47: 598-606.

68. Verma S, Cherayil BJ. Iron and inflammation - the gut reaction. Metallomics: integrated biometal science. 2017; 9: 101-111.

69. Bergamaschi G, Di Sabatino A, Albertini R, Ardizzone S, Biancheri P Bonetti $\mathrm{E}$, et al. Prevalence and pathogenesis of anemia in inflammatory bowel disease. Influence of anti-tumor necrosis factor-alpha treatment. Haematologica. 2010; 95: 199-205

70. David V, Martin A, Isakova T, Spaulding C, Qi L, Ramirez V, et al. Inflammation and functional iron deficiency regulate fibroblast growth factor 23 production. Kidney international. 2016; 89: 135-146.

71. Manghat P, Fraser WD, Wierzbicki AS, Fogelman I, Goldsmith DJ, Hampson G. Fibroblast growth factor-23 is associated with C-reactive protein, serum phosphate and bone mineral density in chronic kidney disease. Osteoporos Int. 2010; 21: 1853-1861.

72. Munoz Mendoza J, Isakova T, Ricardo AC, Xie H, Navaneethan SD, Anderson $\mathrm{AH}$, et al. Fibroblast growth factor 23 and Inflammation in CKD. Clin J Am Soc Nephrol. 2012; 7: 1155-1162.

73. Durlacher-Betzer K, Hassan A, Levi R, Axelrod J, Silver J, Naveh-Many T. Interleukin- 6 contributes to the increase in fibroblast growth factor 23 expression in acute and chronic kidney disease. Kidney Int. 2018; 94: 315325.

74. Ito N, Wijenayaka AR, Prideaux M, Kogawa M, Ormsby RT, Evdokiou A et al. Regulation of FGF23 expression in IDG-SW3 osteocytes and human bone by pro-inflammatory stimuli. Mol Cell Endocrinol. 2015; 399: 208-218.

75. Caza M, Kronstad JW. Shared and distinct mechanisms of iron acquisition by bacterial and fungal pathogens of humans. Frontiers in cellular and infection microbiology. 2013; 3: 80.

76. Holbein BE, Mira de Orduna R. Effect of trace iron levels and iron withdrawa by chelation on the growth of Candida albicans and Candida vini. FEMS Microbiol Lett. 2010; 307: 19-24.

77. Robins-Browne RM, Prpic JK. Effects of iron and desferrioxamine on infections with Yersinia enterocolitica. Infect Immun. 1985; 47: 774-779.

78. Thephinlap C, Phisalaphong C, Fucharoen S, Porter JB, Srichairatanakool S. Efficacy of curcuminoids in alleviation of iron overload and lipid peroxidation in thalassemic mice. Med Chem. 2009; 5: 474-482.

79. Kalinowski DS, Richardson DR. Future of toxicology--iron chelators and differing modes of action and toxicity: the changing face of iron chelation therapy. Chem Res Toxicol. 2007; 20: 715-720

80. Gumbau-Brisa R, Ang MTC, Holbein BE, Bierenstiel M. Enhanced Fe(3+) binding through cooperativity of 3-hydroxypyridin-4-one groups within a linear co-polymer: wrapping effect leading to superior antimicrobial activity.
Biometals. 2020; 33: 339-351.

81. Lehmann C, Aali M, Zhou J, Holbein B. Comparison of Treatment Effects of Different Iron Chelators in Experimental Models of Sepsis. Life. 2021; 11: 57

82. Sullivan JL. Is stored iron safe? J Lab Clin Med. 2004; 144: 280-284.

83. Smith JT, Schneider AD, Katchko KM, Yun C, Hsu EL. Environmental Factors Impacting Bone-Relevant Chemokines. Front Endocrinol (Lausanne). 2017; 8: 22

84. Danford CJ, Trivedi HD, Bonder A. Bone Health in Patients With Liver Diseases. J Clin Densitom. 2020; 23: 212-222.

85. Valenti L, Varenna M, Fracanzani AL, Rossi V, Fargion S, Sinigaglia L. Association between iron overload and osteoporosis in patients with hereditary hemochromatosis. Osteoporos Int. 2009; 20: 549-555.

86. Katsumata S, Katsumata-Tsuboi R, Uehara M, Suzuki K. Severe iron deficiency decreases both bone formation and bone resorption in rats. $J$ Nutr. 2009; 139: 238-243.

87. Yang J, Meng X, Dong D, Xue Y, Chen X, Wang S, et al. Iron overload involved in the enhancement of unloading-induced bone loss by hypomagnetic field. Bone. 2018; 114: 235-245

88. Baschant U, Rauner M, Balaian E, Weidner H, Roetto A, Platzbecker U, et al. Wnt5a is a key target for the pro-osteogenic effects of iron chelation on osteoblast progenitors. Haematologica. 2016; 101: 1499-1507.

89. Fu X, Liu G, Halim A, Ju Y, Luo Q, Song AG. Mesenchymal Stem Cell Migration and Tissue Repair. Cells. 2019; 8: 784

90. Chen B, Yan YL, Liu C, Bo L, Li GF, Wang H, et al. Therapeutic effect of deferoxamine on iron overload-induced inhibition of osteogenesis in a zebrafish model. Calcified tissue international. 2014; 94: 353-360.

91. Zhang J, Zhao H, Yao G, Qiao P, Li L, Wu S. Therapeutic potential of iron chelators on osteoporosis and their cellular mechanisms. Biomed Pharmacother. 2021; 137: 111380

92. Zhang W, Xu J, Qiu J, Xing C, Li X, Leng B, et al. Novel and rapid osteoporosis model established in zebrafish using high iron stress. Biochem Biophys Res Commun. 2018; 496: 654-660.

93. Ishii KA, Fumoto $T$, Iwai $K$, Takeshita $S$, Ito $M$, Shimohata $N$, et al Coordination of PGC-1beta and iron uptake in mitochondrial biogenesis and osteoclast activation. Nat Med. 2009; 15: 259-266.

94. Kim BJ, Ahn $\mathrm{SH}$, Bae SJ, Kim EH, Lee $\mathrm{SH}$, Kim HK, et al. Iron overload accelerates bone loss in healthy postmenopausal women and middle-aged men: a 3-year retrospective longitudinal study. J Bone Miner Res. 2012; 27: 2279-2290.

95. Rossi F, Perrotta S, Bellini G, Luongo L, Tortora C, Siniscalco D, et al Iron overload causes osteoporosis in thalassemia major patients through interaction with transient receptor potential vanilloid type 1 (TRPV1) channels. Haematologica. 2014; 99: 1876-1884

96. Li GF, Pan YZ, Sirois P, Li K, Xu YJ. Iron homeostasis in osteoporosis and its clinical implications. Osteoporos Int. 2012; 23: 2403-2408.

97. Sun L, Guo W, Yin C, Zhang S, Qu G, Hou Y, et al. Hepcidin deficiency undermines bone load-bearing capacity through inducing iron overload. Gene. 2014; 543: 161-165

98. Zhang $\mathrm{P}$, Wang S, Wang L, Shan BC, Zhang $\mathrm{H}$, Yang F, et al. Hepcidin is an endogenous protective factor for osteoporosis by reducing iron levels. J Mol Endocrinol. 2018; 60: 297-306.

99. Sato H, Takai C, Kazama JJ, Wakamatsu A, Hasegawa E, Kobayashi D, et al. Serum hepcidin level, iron metabolism and osteoporosis in patients with rheumatoid arthritis. Sci Rep. 2020; 10: 9882.

100. Liu B, Liu C, Zhong W, Song M, Du S, Su J. Reduced hepcidin level features osteoporosis. Exp Ther Med. 2018; 16: 1963-1967.

101. Argenziano M, Di Paola A, Tortora C, Di Pinto D, Pota E, Di Martino M, et al. Effects of Iron Chelation in Osteosarcoma. Curr Cancer Drug Targets. 2020.

102. Forciniti S, Greco L, Grizzi F, Malesci A, Laghi L. Iron Metabolism in Cancer 
Progression. Int J Mol Sci. 2020; 21: 2257.

103. Jung M, Mertens C, Tomat E, Brune B. Iron as a Central Player and Promising Target in Cancer Progression. Int J Mol Sci. 2019; 20: 273.

104. Bystrom LM, Rivella S. Cancer cells with irons in the fire. Free Radic Biol Med. 2015; 79: 337-342.

105. Wang F, Lv H, Zhao B, Zhou L, Wang S, Luo J, et al. Iron and leukemia: new insights for future treatments. J Exp Clin Cancer Res. 2019; 38: 406.

106. Greene CJ, Attwood K, Sharma NJ, Gross KW, Smith GJ, Xu B, et al. Transferrin receptor 1 upregulation in primary tumor and downregulation in benign kidney is associated with progression and mortality in renal cell carcinoma patients. Oncotarget. 2017; 8: 107052-107075.

107. Jeong SM, Hwang S, Seong RH. Transferrin receptor regulates pancreatic cancer growth by modulating mitochondrial respiration and ROS generation. Biochem Biophys Res Commun. 2016; 471: 373-379.

108. Manz DH, Blanchette NL, Paul BT, Torti FM, Torti SV. Iron and cancer: recent insights. Annals of the New York Academy of Sciences. 2016; 1368 149-161.

109.Bauckman K, Haller E, Taran N, Rockfield S, Ruiz-Rivera A, Nanjundan $M$. Iron alters cell survival in a mitochondria-dependent pathway in ovarian cancer cells. Biochem J. 2015; 466: 401-413.

110. Wang SL, Cao S, Wu R, Chi F, Tang MY, Jin XY, et al. Serum ferritin predicted prognosis in patients with locally advanced pancreatic cancer Future Oncol. 2015; 11: 2905-2910.

111. Wang X, An P, Zeng J, Liu X, Wang B, Fang X, et al. Serum ferritin in combination with prostate-specific antigen improves predictive accuracy for prostate cancer. Oncotarget. 2017; 8: 17862-17872.

112. Lebon D, Vergez F, Bertoli S, Harrivel V, De Botton S, Micol JB, et al. Hyperferritinemia at diagnosis predicts relapse and overall survival in younger AML patients with intermediate-risk cytogenetics. Leuk Res. 2015; 39: 818-821.

113. Pinnix ZK, Miller LD, Wang W, D'Agostino R, Jr., Kute T, Willingham MC, et al. Ferroportin and iron regulation in breast cancer progression and prognosis. Sci Transl Med. 2010; 2: 43ra56.

114. Trujillo-Alonso V, Pratt EC, Zong H, Lara-Martinez A, Kaittanis C, Rabie MO, et al. FDA-approved ferumoxytol displays anti-leukaemia efficacy against cells with low ferroportin levels. Nat Nanotechnol. 2019; 14 616-622.

115. Gasparetto M, Pei S, Minhajuddin M, Stevens B, Smith CA, Seligman P. Low ferroportin expression in AML is correlated with good risk cytogenetics, improved outcomes and increased sensitivity to chemotherapy. Leuk Res. 2019; 80: 1-10.

116. Darshan D, Anderson GJ. Interacting signals in the control of hepcidin expression. Biometals. 2009; 22: 77-87.

117. Ganz T. Hepcidin, a key regulator of iron metabolism and mediator of anemia of inflammation. Blood. 2003; 102: 783-788.

118. Nemeth E, Ganz T. The role of hepcidin in iron metabolism. Acta Haematol. 2009; 122: 78-86.

119. Sasu BJ, Li H, Rose MJ, Arvedson TL, Doellgast G, Molineux G. Serum hepcidin but not prohepcidin may be an effective marker for anemia of inflammation (AI). Blood Cells Mol Dis. 2010; 45: 238-245.

120. Lamy PJ, Durigova A, Jacot W. Iron homeostasis and anemia markers in early breast cancer. Clin Chim Acta. 2014; 434: 34-40.

121. Mei S, Wang H, Fu R, Qu W, Xing L, Wang G, et al. Hepcidin and GDF15 in anemia of multiple myeloma. Int J Hematol. 2014; 100: 266-273.

122. Tanno T, Rabel A, Alleyne M, Lee YT, Dahut WL, Gulley JL, et al. Hepcidin, anaemia, and prostate cancer. BJU Int. 2011; 107: 678-679.

123. Tesfay L, Clausen KA, Kim JW, Hegde P, Wang X, Miller LD, et al. Hepcidin regulation in prostate and its disruption in prostate cancer. Cancer Res. 2015; 75: 2254-2263.

124. Kamai T, Tomosugi N, Abe H, Arai K, Yoshida K. Increased serum hepcidin-25 level and increased tumor expression of hepcidin mRNA are associated with metastasis of renal cell carcinoma. BMC Cancer. 2009; 9 : 270.

125. Fischbacher A, von Sonntag C, Schmidt TC. Hydroxyl radical yields in the Fenton process under various $\mathrm{pH}$, ligand concentrations and hydrogen peroxide/Fe(II) ratios. Chemosphere. 2017; 182: 738-744.

126. Ibrahim O, O'Sullivan J. Iron chelators in cancer therapy. Biometals. 2020; 33: $201-215$

127. Jeon SR, Lee JW, Jang PS, Chung NG, Cho B, Jeong DC. Anti-leukemic properties of deferasirox via apoptosis in murine leukemia cell lines. Blood Res. 2015; 50: 33-39.

128. Shinoda S, Kaino S, Amano S, Harima H, Matsumoto T, Fujisawa K, et al Deferasirox, an oral iron chelator, with gemcitabine synergistically inhibits pancreatic cancer cell growth in vitro and in vivo. Oncotarget. 2018; 9: 28434-28444.

129. Yang $Y, X u Y$, Su A, Yang D, Zhang X. Effects of Deferoxamine on Leukemia In Vitro and Its Related Mechanism. Med Sci Monit. 2018; 24: 6735-6741.

130. Vela D. Iron in the Tumor Microenvironment. Adv Exp Med Biol. 2020; 1259 39-51.

131. Arneth B. Tumor Microenvironment. Medicina (Kaunas). 2019; 56.

132. Punzo F, Bellini G, Tortora C, Pinto DD, Argenziano M, Pota E, et al. Mifamurtide and TAM-like macrophages: effect on proliferation, migration and differentiation of osteosarcoma cells. Oncotarget. 2020; 11: 687-698.

133. Van Overmeire E, Laoui D, Keirsse J, Van Ginderachter JA, Sarukhan A. Mechanisms driving macrophage diversity and specialization in distinct tumor microenvironments and parallelisms with other tissues. Front Immunol. 2014; 5: 127

134. Recalcati S, Locati M, Marini A, Santambrogio P, Zaninotto F, De Pizzol M et al. Differential regulation of iron homeostasis during human macrophage polarized activation. Eur J Immunol. 2010; 40: 824-835.

135. Lane DJ, Merlot AM, Huang ML, Bae DH, Jansson PJ, Sahni S, et al. Cellular iron uptake, trafficking and metabolism: Key molecules and mechanisms and their roles in disease. Biochim Biophys Acta. 2015; 1853: 1130-1144.

136. Brown RAM, Richardson KL, Kabir TD, Trinder D, Ganss R, Leedman PJ. Altered Iron Metabolism and Impact in Cancer Biology, Metastasis, and Immunology. Front Oncol. 2020; 10: 476.

137. Kim DH, Kim JH, Kim EH, Na HK, Cha YN, Chung JH, et al. 15-DeoxyDelta12,14-prostaglandin J2 upregulates the expression of heme oxygenase- 1 and subsequently matrix metalloproteinase- 1 in human breast cancer cells: possible roles of iron and ROS. Carcinogenesis. 2009; 30: 645654.

138. Luanpitpong S, Talbott SJ, Rojanasakul Y, Nimmannit U, Pongrakhananon $\mathrm{V}$, Wang $\mathrm{L}$, et al. Regulation of lung cancer cell migration and invasion by reactive oxygen species and caveolin-1. J Biol Chem. 2010; 285: 3883238840 .

139. Zhao B, Li R, Cheng G, Li Z, Zhang Z, Li J, et al. Role of hepcidin and iron metabolism in the onset of prostate cancer. Oncol Lett. 2018; 15: 9953-9958.

140. Kaomongkolgit R, Cheepsunthorn P, Pavasant P, Sanchavanakit N. Iron increases MMP-9 expression through activation of AP-1 via ERK/Akt pathway in human head and neck squamous carcinoma cells. Oral Oncol. 2008; 44: 587-594.

141. Yu Y, Gutierrez E, Kovacevic Z, Saletta F, Obeidy P, Suryo Rahmanto Y et al. Iron chelators for the treatment of cancer. Curr Med Chem. 2012; 19 : 2689-2702.

142. Netz DJ, Stith CM, Stumpfig M, Kopf G, Vogel D, Genau HM, et al. Eukaryotic DNA polymerases require an iron-sulfur cluster for the formation of active complexes. Nat Chem Biol. 2011; 8: 125-132.

143. Malumbres M, Barbacid M. Cell cycle, CDKs and cancer: a changing paradigm. Nat Rev Cancer. 2009; 9: 153-166.

144. Kulp KS, Green SL, Vulliet PR. Iron deprivation inhibits cyclin-dependent 
kinase activity and decreases cyclin D/CDK4 protein levels in asynchronous MDA-MB-453 human breast cancer cells. Exp Cell Res. 1996; 229: 60-68.

145. Brahimi-Horn MC, Chiche J, Pouyssegur J. Hypoxia and cancer. J Mol Med (Berl). 2007; 85: 1301-1307.

146. Keith B, Johnson RS, Simon MC. HIF1alpha and HIF2alpha: sibling rivalry in hypoxic tumour growth and progression. Nat Rev Cancer. 2011; 12: 9-22.

147. Renassia C, Peyssonnaux C. New insights into the links between hypoxia and iron homeostasis. Curr Opin Hematol. 2019; 26: 125-130.

148. Xue X, Taylor M, Anderson E, Hao C, Qu A, Greenson JK, et al. Hypoxiainducible factor-2alpha activation promotes colorectal cancer progression by dysregulating iron homeostasis. Cancer Res. 2012; 72: 2285-2293.

149. Le NT, Richardson DR. The role of iron in cell cycle progression and the proliferation of neoplastic cells. Biochim Biophys Acta. 2002; 1603: 31-46.

150. Richardson DR. Therapeutic potential of iron chelators in cancer therapy. Adv Exp Med Biol. 2002; 509: 231-249

151.Donfrancesco A, De Bernardi B, Carli M, Mancini A, Nigro M, De Sio L, et al. Deferoxamine followed by cyclophosphamide, etoposide, carboplatin, thiotepa, induction regimen in advanced neuroblastoma: preliminary results. Italian Neuroblastoma Cooperative Group. Eur J Cancer. 1995; 31A: 612 615.

152. Porter JB, Evangeli M, El-Beshlawy A. Challenges of adherence and persistence with iron chelation therapy. Int J Hematol. 2011; 94: 453-460.

153. Choi JH, Kim JS, Won YW, Uhm J, Park BB, Lee YY. The potential of deferasirox as a novel therapeutic modality in gastric cancer. World J Surg Oncol. 2016; 14: 77

154. Amano S, Kaino S, Shinoda S, Harima H, Matsumoto T, Fujisawa K, et al. Invasion inhibition in pancreatic cancer using the oral iron chelating agent deferasirox. BMC Cancer. 2020; 20: 681

155. Bendova P, Mackova E, Haskova P, Vavrova A, Jirkovsky E, Sterba M, et al. Comparison of clinically used and experimental iron chelators for protection against oxidative stress-induced cellular injury. Chem Res Toxicol. 2010 23: $1105-1114$

156. Callens C, Coulon S, Naudin J, Radford-Weiss I, Boissel N, Raffoux E, et al. Targeting iron homeostasis induces cellular differentiation and synergizes with differentiating agents in acute myeloid leukemia. J Exp Med. 2010; 207: 731-750.
157. Krishan S, Sahni S, Richardson DR. The anti-tumor agent, Dp44mT, promotes nuclear translocation of TFEB via inhibition of the AMPK-mTORC1 axis. Biochim Biophys Acta Mol Basis Dis. 2020; 1866: 165970.

158. Zhou J, Jiang $\mathrm{Y}$, Zhao J, Zhang $\mathrm{H}$, Fu J, Luo P, et al. Dp44mT, an iron chelator, suppresses growth and induces apoptosis via RORA-mediated NDRG2-IL6/JAK2/STAT3 signaling in glioma. Cell Oncol (Dordr). 2020; 43 $461-475$.

159. Zou Y, Zhou J, Xu B, Li W, Wang Z. Ribonucleotide reductase subunit M2 as a novel target for clear-cell renal cell carcinoma. Onco Targets Ther. 2019 12: 3267-3275.

160.Zeidner JF, Karp JE, Blackford AL, Smith BD, Gojo I, Gore SD, et al. A phase II trial of sequential ribonucleotide reductase inhibition in aggressive myeloproliferative neoplasms. Haematologica. 2014; 99: 672-678.

161. Fryknas M, Zhang X, Bremberg $U$, Senkowski W, Olofsson MH, Brandt $P$ et al. Iron chelators target both proliferating and quiescent cancer cells. Sci Rep. 2016; 6: 38343

162. Gan RY, Li HB, Sui ZQ, Corke H. Absorption, metabolism, anti-cancer effect and molecular targets of epigallocatechin gallate (EGCG): An updated review. Crit Rev Food Sci Nutr. 2018; 58: 924-941.

163. Rauf A, Imran M, Khan IA, Ur-Rehman M, Gilani SA, Mehmood Z, et al. Anticancer potential of quercetin: A comprehensive review. Phytother Res. 2018; 32: 2109-2130.

164. Ting H, Deep G, Agarwal R. Molecular mechanisms of silibinin-mediated cancer chemoprevention with major emphasis on prostate cancer. AAPS J. 2013; 15: 707-716.

165. Tury S, Assayag F, Bonin F, Chateau-Joubert S, Servely JL, Vacher S, et al. The iron chelator deferasirox synergises with chemotherapy to treat triplenegative breast cancers. J Pathol. 2018; 246: 103-114.

166. Piro E, Lentini M, Levato L, Russo A, Molica S. Sustained Erythroid Response in a Patient with Myelofibrosis Receiving Concomitant Treatment with Ruxolitinib and Deferasirox. Chemotherapy. 2018; 63: 107-110. 